\title{
Evidence for Time-Reversal Symmetry Breaking of the Superconducting State near Twin-Boundary Interfaces in FeSe Revealed by Scanning Tunneling Spectroscopy
}

\author{
T. Watashige, ${ }^{1,2}$ Y. Tsutsumi, ${ }^{3,}{ }^{*}$ T. Hanaguri, ${ }^{2}$ Y. Kohsaka, ${ }^{2}$ S. Kasahara, ${ }^{1}$ A. Furusaki, ${ }^{2,3}$ M. Sigrist, ${ }^{4}$ \\ C. Meingast, ${ }^{5}$ T. Wolf, ${ }^{5}$ H. v. Löhneysen, ${ }^{5}$ T. Shibauchi, ${ }^{6,1}$ and Y. Matsuda ${ }^{1}$ \\ ${ }^{1}$ Department of Physics, Kyoto University, Kyoto 606-8502, Japan \\ ${ }^{2}$ RIKEN Center for Emergent Matter Science, Wako, Saitama 351-0198, Japan \\ ${ }^{3}$ Condensed Matter Theory Laboratory, RIKEN, Wako, Saitama 351-0198, Japan \\ ${ }^{4}$ Theoretische Physik, ETH Zürich, CH-8093 Zürich, Switzerland \\ ${ }^{5}$ Institute of Solid State Physics (IFP), Karlsruhe Institute of Technology, D-76021 Karlsruhe, Germany \\ ${ }^{6}$ Department of Advanced Materials Science, University of Tokyo, Chiba 277-8561, Japan \\ (Received 12 March 2015; revised manuscript received 21 June 2015; published 21 August 2015)
}

\begin{abstract}
Junctions and interfaces consisting of unconventional superconductors provide an excellent experimental playground to study exotic phenomena related to the phase of the order parameter. Not only does the complex structure of unconventional order parameters have an impact on the Josephson effects, but it also may profoundly alter the quasiparticle excitation spectrum near a junction. Here, by using spectroscopic-imaging scanning tunneling microscopy, we visualize the spatial evolution of the LDOS near twin boundaries (TBs) of the nodal superconductor FeSe. The $\pi / 2$ rotation of the crystallographic orientation across the TB twists the structure of the unconventional order parameter, which may, in principle, bring about a zero-energy LDOS peak at the TB. The LDOS at the TB observed in our study, in contrast, does not exhibit any signature of a zero-energy peak, and an apparent gap amplitude remains finite all the way across the TB. The low-energy quasiparticle excitations associated with the gap nodes are affected by the TB over a distance more than an order of magnitude larger than the coherence length $\xi_{a b}$. The modification of the low-energy states is even more prominent in the region between two neighboring TBs separated by a distance $\approx 7 \xi_{a b}$. In this region, the spectral weight near the Fermi level $(\approx \pm 0.2 \mathrm{meV})$ due to the nodal quasiparticle spectrum is almost completely removed. These behaviors suggest that the TB induces a fully gapped state, invoking a possible twist of the order parameter structure, which breaks time-reversal symmetry.
\end{abstract}

DOI: 10.1103/PhysRevX.5.031022

\section{INTRODUCTION}

When two superconductors are in close proximity, they are influenced by each other via the tunneling of Cooper pairs. The Cooper pair tunneling results in the flow of a superconducting Josephson current, which has been studied for decades and is used in various superconducting quantum devices [1]. The Josephson current is governed by the phase difference of the order parameters of the two superconductors. Therefore, Josephson junctions consisting of unconventional superconductors, where the superconducting order parameter changes its sign depending on the momentum direction, serve as a unique platform where novel phase-related phenomena, e.g., spontaneous

*Present address: Department of Basic Science, The University of Tokyo, 3-8-1 Komaba, Meguro-ku, Tokyo 153-8902, Japan.

Published by the American Physical Society under the terms of the Creative Commons Attribution 3.0 License. Further distribution of this work must maintain attribution to the author(s) and the published article's title, journal citation, and DOI.
Subject Areas: Condensed Matter Physics,

Superconductivity

formation of half-flux quanta in a trijunction of cuprate superconductors [2], take place. Compared to the wellinvestigated Josephson currents, the spatial and energy dependence of the superconducting order parameter and quasiparticle states around these junctions remain to be understood.

Recent progress in STM and STS technologies opens up a way to directly visualize the spatial variation of the electronic states in superconducting heterostructures [3-5]. However, STM or STS studies on superconducting junctions made of unconventional superconductors are still demanding. There are two reasons that make it difficult to study unconventional junctions. First, it is often challenging to artificially fabricate well-defined junctions of unconventional superconductors. Second, in most unconventional superconductors, surfaces are not electronically neutral; the resultant charge accumulation at the surfaces prevents STM or STS from accessing bulk superconducting properties. In this study, we solve these problems by inspecting the twin boundaries (TBs) in the nodal ironbased superconductor FeSe [6,7]. 
The TB is a crystallographic plane in a crystal shared by two neighboring domains with one being the mirror image of the other. The TBs are often formed by a tetragonal-toorthorhombic structural phase transition, which reduces the fourfold $\left(C_{4}\right)$ symmetry at high temperature to twofold $\left(C_{2}\right)$ symmetry at low temperature. In such a case, the orthorhombic crystal may contain the TBs parallel to the (110) plane, which act as an atomically well-defined junction. Some unconventional-superconductor-related materials, such as $\mathrm{YBa}_{2} \mathrm{Cu}_{3} \mathrm{O}_{7-\delta}, A E\left(\mathrm{Fe}_{1-x} \mathrm{Co}_{x}\right)_{2} \mathrm{As}_{2}$ (where $A E$ is alkali-earth element) and $\mathrm{NaFeAs}$, do form TBs upon the tetragonal-to-orthorhombic transition that were identified by STM or STS measurements [8-10]. However, unavoidable surface state formation and/or an insufficient amount of chemical doping prevent the STM or STS measurements from accessing superconductivity near TBs in these materials.

FeSe (superconducting transition temperature $T_{c} \approx 9 \mathrm{~K}$ [11]) is a promising candidate for studying the effects of TBs on unconventional superconductivity by STM or STS. Among various iron-based superconductors, FeSe has the simplest crystal structure [Fig. 1(a)] in which electronically neutral two-dimensional FeSe layers are stacked along the $c$ axis [11]. This guarantees the perfect cleaved surface, which is electronically neutral. The tetragonal-to-orthorhombic structural phase transition, which is likely caused by the orbital ordering [12-17], occurs at $T_{s} \approx 90 \mathrm{~K}$ and the TBs are spontaneously formed in the orthorhombic phase as illustrated in Fig. 1(b).

Band-structure calculations show that the Fermi surface of FeSe consists of hole cylinders around the zone center and compensating electron cylinders around the zone corner $[19,20]$. Several measurements, including penetration depth, quasiparticle interference, thermoelectric response [7], quantum oscillations [14,21,22], and angleresolved photoemission spectroscopy (ARPES) [12-15,23] reveal that the Fermi surface in the orthorhombic phase consists of one hole and one (or two [14,22]) electron bands, both of which have very low carrier densities. The V-shaped tunneling spectrum [6], quasilinear temperature dependence of the penetration depth down to $80 \mathrm{mK}$ [7], and the sizable residual thermal conductivity at $T \rightarrow 0$ in clean crystals [7] all provide strong evidence that FeSe is an unconventional superconductor with line nodes in the superconducting gap.

The TBs in FeSe have been studied by low-temperature (4.2 K) STM or STS in the films grown by molecular beam epitaxy, and the suppression of superconductivity by the
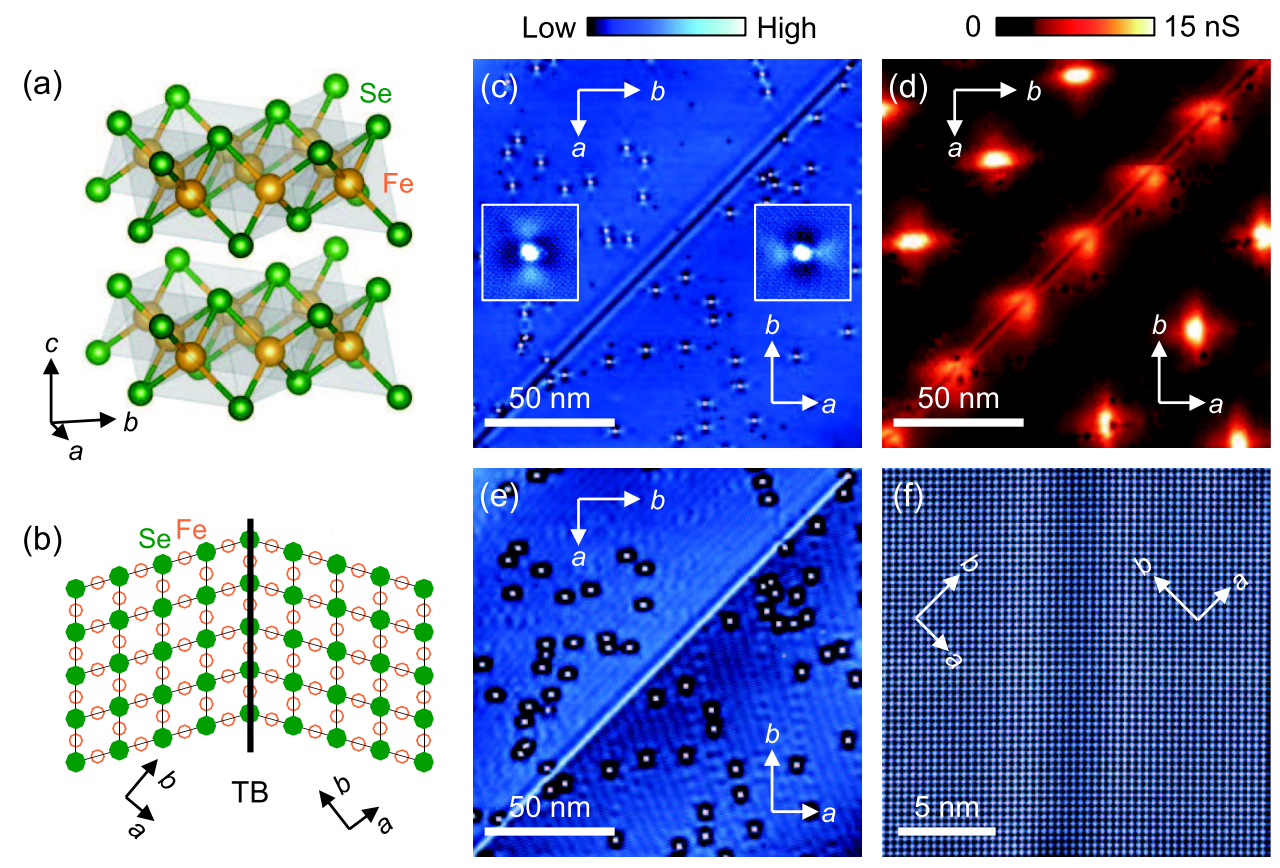

FIG. 1. (a) Crystal structure of FeSe visualized using the VESTA program [18]. (b) Schematic top view of the atomic arrangement near the TB of FeSe (not in scale). Green filled circles and orange open circles denote topmost Se and Fe atoms, respectively. Se atoms beneath the Fe layer are not shown. (c) A constant-current STM image of the cleaved (001) surface of FeSe at $1.5 \mathrm{~K}$ showing the TB running from bottom left to top right. Crystallographic axes parallel to the Fe-Fe direction are shown by white arrows $(b>a)$. The two insets show a magnified image of the defect $\left(8.8 \times 8.8 \mathrm{~nm}^{2}\right)$ in the upper-left or lower-right domain. Note that the pattern is rotated by $\pi / 2$ between the two domains. The setup conditions for imaging are $V_{s}=+95 \mathrm{mV}$ and $I_{t}=10 \mathrm{pA}$. (d) Zero-bias conductance image $g(\boldsymbol{r}, E=0)$ at $1.5 \mathrm{~K}$ showing vortices. A magnetic field of $1 \mathrm{~T}$ is applied along the $c$ axis. The tip is stabilized at $V_{s}=+10 \mathrm{mV}$ and $I_{t}=100 \mathrm{pA}$. A bias modulation amplitude $V_{\text {mod }}=0.21 \mathrm{mV}_{\mathrm{rms}}$ is used for spectroscopy. (e) A low-bias STM image at $1.5 \mathrm{~K}$ taken with $V_{s}=+20 \mathrm{mV}$ and $I_{t}=10 \mathrm{pA}$. The field of view for (c)-(e) is the same. (f) An atomic-resolution STM image near the TB, which is running vertically in the center of the field of view. $V_{s}=+95 \mathrm{mV}$ and $I_{t}=100 \mathrm{pA}$. 
TBs has been reported [24]. We perform STM or STS measurements at much lower temperature $(\approx 0.4 \mathrm{~K})$ in vapor-grown single crystals to examine the details of the superconducting gap and quasiparticle excitations near the TBs.

\section{EXPERIMENTAL METHOD}

STM or STS experiments are conducted in a constantcurrent mode with a commercial ultrahigh vacuum very low temperature STM (UNISOKU, USM-1300) modified by ourselves [25]. The samples used in this study are highquality bulk single crystals grown using the vapor transport method [26]. Superconducting transition temperature defined at zero resistance is about $9 \mathrm{~K}$. These crystals are undoped and stoichiometric, enabling us to investigate uniform and clean TBs. Samples are cleaved in situ at liquid $\mathrm{N}_{2}$ temperature to prepare clean and flat (001) surfaces. Immediately after cleaving, the samples are transferred to the STM unit kept below $10 \mathrm{~K}$. We use electrochemically etched polycrystalline tungsten wires for the scanning tips, which were cleaned and sharpened in situ by field evaporation using field-ion microscopy. The tunneling conductance $g(\boldsymbol{r}, E) \equiv d I_{t} / d V_{s}(\boldsymbol{r}, E)$ reflecting the LDOS at a position $\boldsymbol{r}$ and energy $E$ is acquired by the standard lock-in technique. Here, $I_{t}$ and $V_{s}$ denote the tunneling current and the sample-bias voltage, respectively.

\section{RESULTS AND DISCUSSION}

\section{A. Imaging the twin boundary in FeSe}

Figure 1(c) depicts a STM image of the cleaved surface of an FeSe single crystal at temperature $T=1.5 \mathrm{~K}$. The image demonstrates the extremely small concentration of defects, i.e., about one defect per $5000 \mathrm{Fe}$ atoms in the (001) plane. There is a shallow "groove" running along the [110] direction of the Fe lattice across which the unidirectional feature around the point defect is rotated by $\pi / 2$, indicating that the groove represents the TB. We also observe that the elongated vortex cores [6], which are imaged by mapping $g(\boldsymbol{r}, E=0)$ in a magnetic field, are rotated by $\pi / 2$ across the TB [Fig. 1(d)]. What is intriguing is that the vortices trapped at the TB are not elongated along the $\mathrm{TB}$, demonstrating that the critical current density across the TB is comparable to that of the bulk. The STM image of the TB at a lower bias voltage is not a groove but a "ridge" [Fig. 1(e)]. This suggests that the apparent corrugations near the TB are primarily associated with the electronic-state variations; the actual surface topography near the TB may be essentially flat. A magnified STM image near the TB is shown in Fig. 1(f). A regular square lattice of the topmost Se atoms is well maintained even in the close vicinity of the TB. These observations indicate that the TB in FeSe is an atomically sharp superconducting junction with minimal strain to the lattice. (A detailed argument regarding the absence of strain is given in Appendix A.)

\section{B. Local density of states across the twin boundaries}

We examine the LDOS evolution across the TB by taking a series of $g(\boldsymbol{r}, E)$ along the line indicated in Fig. 2(a). Here and in the following, we are interested only in the evolution of $g(\boldsymbol{r}, E)$ along the $x$ axis running perpendicular to the TB leaving the $y$ coordinate constant, hence, $g(x, E)$. Figure 2(b) shows an intensity plot of $g(\boldsymbol{r}, E)$. Individual spectra taken at representative points are depicted in Fig. 2(c). At the position far away from the TB (I), $g(\boldsymbol{r}, E)$ exhibits a superconducting gap with clear quasiparticle peaks at $\approx \pm 2.5 \mathrm{meV}$. In addition to this main feature, there is a shoulder outside of the main peaks $(\approx \pm 3.5 \mathrm{meV})$, which may represent multiple superconducting gaps [7]. In contrast to the case of fully gapped superconductors in which $g(\boldsymbol{r}, E)=0$ in an extended $E$ region near $E=0, g(\boldsymbol{r}, E)$ in FeSe approaches zero only for $E \rightarrow 0$ and is apparently $\mathrm{V}$-shaped, indicating the presence of line nodes [6]. Even right at the TB (III), the residual LDOS at $E=0$ is negligibly small, indicating that the TB hardly gives rise to a pair-breaking effect. In the vicinity of the TB, the quasiparticle peak and the shoulder associated with the superconducting gap diminish, and instead, sharp particle-hole symmetric peaks appear at $E \approx \pm 1.5 \mathrm{meV}$. It is important to note that the spatial evolution of the spectrum does not accompany the smooth change in the quasiparticlepeak energy; instead, the $1.5-\mathrm{meV}$ peak coexists with the $2.5-\mathrm{meV}$ peak in the crossover region (II). This means that the $1.5-\mathrm{meV}$ peak is not caused by the suppression of the superconducting gap due to the TB but represents the bound state generated by the TB. The $1.5-\mathrm{meV}$ peak is observed over about $5 \mathrm{~nm}$ from the TB, which is close to the "averaged" in-plane coherence length $\xi_{a b} \approx 5 \mathrm{~nm}$ obtained from the upper critical field $H_{c 2}(\| c) \approx 15 \mathrm{~T}[7,21]$.

Another interesting observation is that low-energy quasiparticle excitations are suppressed over a very long distance from the TB. High-resolution $g(\boldsymbol{r}, E)$ spectra at the positions of (I), (II), and (III) are plotted in Fig. 2(d). While the overall V-shaped behavior is maintained, the exact shape near the bottom of the gap depends on the position. In order to examine this behavior, we fit an empirical power law $g(\boldsymbol{r}, E) \propto|E|^{\alpha}$ to the low-energy $(|E|<0.5 \mathrm{meV})$ spectra and plot the exponent $\alpha$ as a function of the distance from the TB at $x=0$ [Fig. 2(e)]. Except close to the TB $\left(|x| \lesssim \xi_{a b}\right)$, where the 1.5 -meV peaks dominate, $\alpha$ increases gradually with decreasing $x$ by about $\approx 40 \%$. This implies the suppression of the low-energy quasiparticle excitations, most probably due to the opening of a small gap induced by the TB. The salient feature is that $\alpha$ continues to change even at $|x|>10 \xi_{a b}(\approx 50 \mathrm{~nm})$, indicating an unexpectedly long-distance influence of the TB.

The long-distance TB effect on the LDOS can be seen in a more dramatic way in two junctions in series formed by 

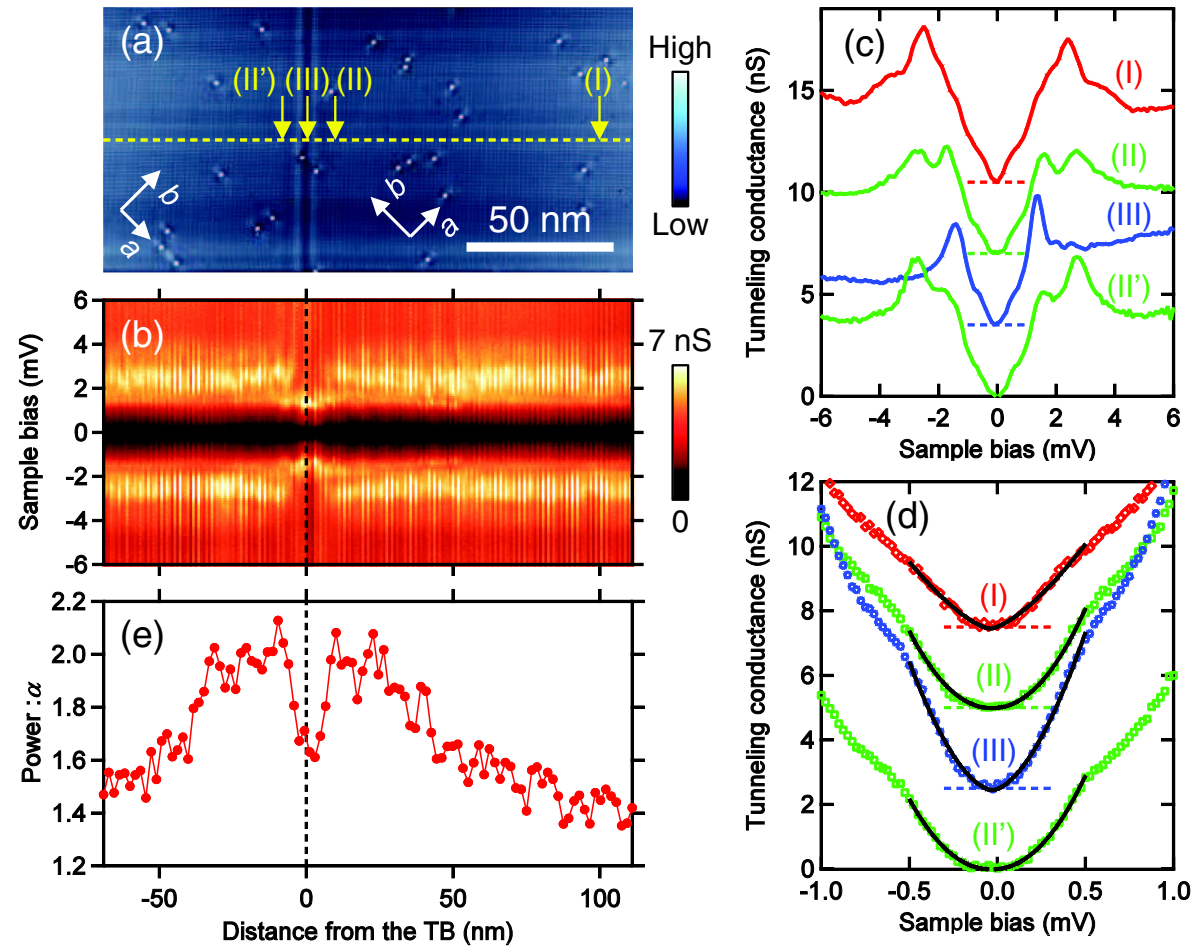

FIG. 2. (a) A constant-current STM image at $1.5 \mathrm{~K}$ near a TB taken at $V_{s}=+95 \mathrm{mV}$ and $I_{t}=100$ pA. (b) Intensity plot of $g(\boldsymbol{r}, E)$ along the yellow broken line in (a). $V_{s}=+20 \mathrm{mV}, I_{t}=100 \mathrm{pA}$, and $V_{\text {mod }}=0.05 \mathrm{mV}_{\mathrm{rms}}$. Spectroscopic measurements are done at $0.4 \mathrm{~K}$. (c) Tunneling spectra at the representative points indicated in (a). Positions (II) and (II') are symmetric about the TB. $V_{s}=+20 \mathrm{mV}, I_{t}=100 \mathrm{pA}$, and $V_{\text {mod }}=0.05 \mathrm{mV}_{\mathrm{rms}}$. (d) High-resolution tunneling spectra at low $E$ taken at the same positions as for (c). $V_{s}=+10 \mathrm{mV}, I_{t}=100 \mathrm{pA}$, and $V_{\text {mod }}=0.025 \mathrm{mV}_{\mathrm{rms}}$. Open symbols and solid lines denote experimental data and fitted results, respectively. Apparent kinks are observed at $\approx \pm 0.5 \mathrm{mV}$ regardless of the positions. The origin of these features is unclear. The fitting is made below this energy scale. Spectra shown in (c) and (d) are shifted vertically for clarity. (e) The exponent $\alpha\left[g(\boldsymbol{r}, E) \propto|E|^{\alpha}\right]$ determined from the fit to the experimental data in the range of $|E| \leq 0.5 \mathrm{meV}$ plotted as a function of the distance from the TB.

two TBs. As shown in Fig. 3(a), we find an area where two TBs are running parallel to each other. The distance between the TBs is $34 \mathrm{~nm}$, which is about 7 times larger than $\xi_{a b}$. Figure 3(b) shows the spatial evolution of $g(\boldsymbol{r}, E)$ across the double TBs. Individual spectra at representative points are plotted in Fig. 3(c). The overall spectral features, the $2.5-\mathrm{meV}$ peak, the $3.5-\mathrm{meV}$ shoulder, and the $1.5-\mathrm{meV}$ peak observed near a single TB are all reproduced (positions I, II, and III). However, the low-energy spectrum taken inside the central domain (position IV) shows a striking anomaly that is absent in the case of a single TB. Figure 3(d) depicts $g(\boldsymbol{r}, E)$ spectra at low energies. It is clear that, in between the double TBs, there is a finite energy range where $g(\boldsymbol{r}, E)$ is almost completely zero. The noticeable difference of the gap structure between inside and outside the central domain is clearly seen in Fig. 3(e), which shows the exponent $\alpha$ plotted as a function of the distance from one of the TBs; $\alpha$ is strongly enhanced in the central domain and peaks at the middle of the domain. The large power $\alpha \approx 4$, which is $\approx 3$ times larger than the values at large $x$, is essentially indistinguishable from an exponential energy dependence. This apparent large power again corroborates the finite gap opening in the excitation spectrum of the quasiparticle.

\section{Possible time-reversal-symmetry-broken state near the twin boundary}

The above observations, the TB-induced bound states at finite energies and the suppression of the low-energy quasiparticle excitations over a length scale much longer than $\xi_{a b}$, suggest a novel role of the TB in an unconventional superconductor. In order to understand the origin of these anomalies, it is important to examine the structure of the gap node in momentum space. We note that the so-called horizontal node [27-29] is unlikely in FeSe. If it exists, the horizontal node is on a hole cylinder and requires strong dispersion along the $c$ axis associated with the mixing of the $d_{z^{2}}$ orbital to the band with $d_{x z}$ or $d_{y z}$ character near the $Z$ point [27-29]. However, recent a ARPES experiment has revealed that the hole cylinder is essentially two dimensional even though there is a finite dispersion along the $c$ axis; its orbital character keeps $d_{x z}$ or $d_{y z}$ everywhere [14]. Moreover, if the horizontal node were the case, there would be no way to alter the superconducting gap across the TB, thereby nodal excitations should not be affected at all. This is apparently contradictory to the suppression of low-energy quasiparticle excitations observed over long distances from the TBs [Figs. 2(e) and 3(e)]. 

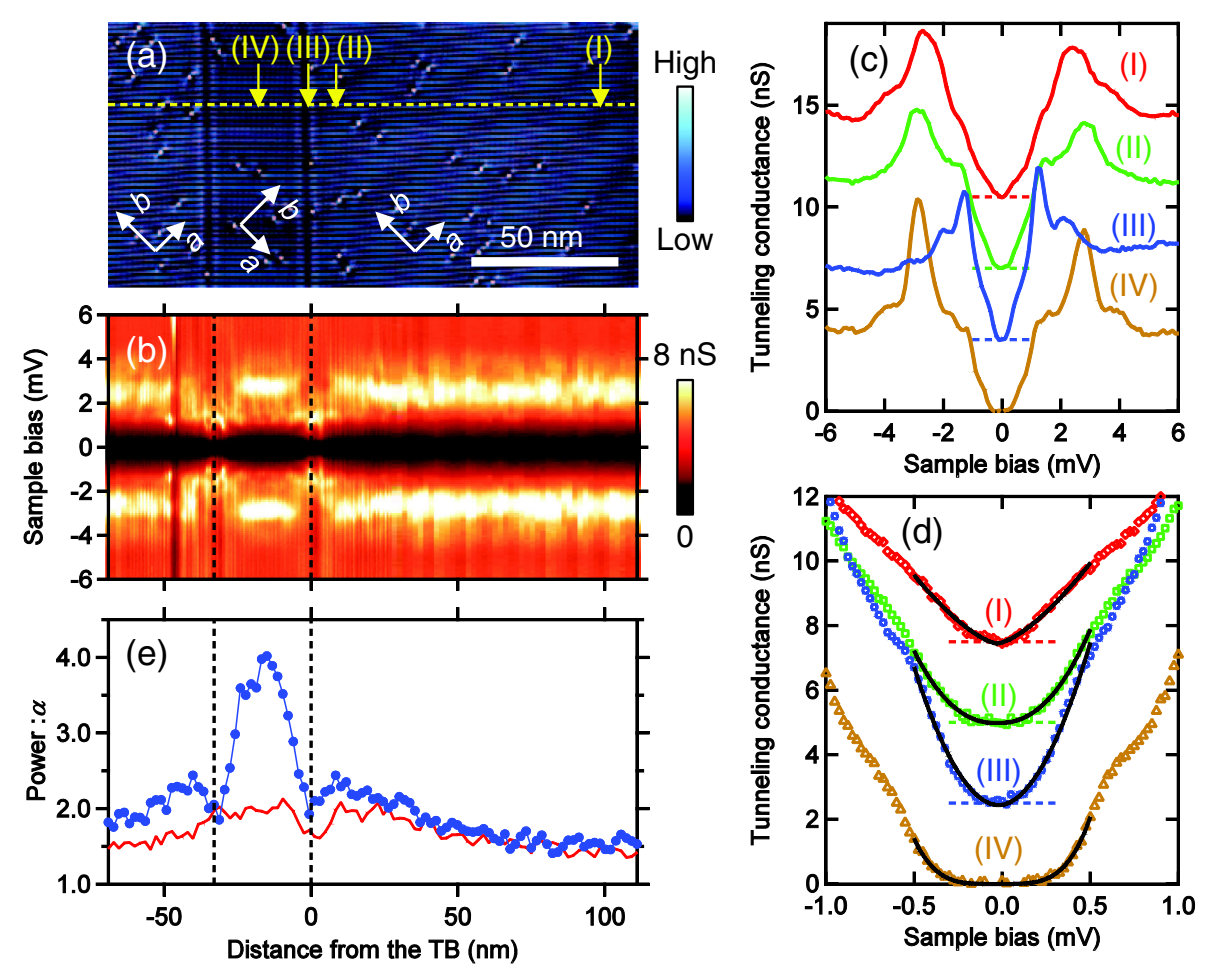

FIG. 3. (a) Constant-current STM image at $1.5 \mathrm{~K}$ with double TBs taken at $V_{s}=+95 \mathrm{mV}$ and $I_{t}=10 \mathrm{pA}$. (b) Intensity plot of $g(\boldsymbol{r}, E)$ along the yellow broken line in (a). Positions of the TBs are indicated by broken lines. A low-conductance position at $\approx-47 \mathrm{~nm}$ is due to a point defect nearby. $V_{s}=+20 \mathrm{mV}, I_{t}=100 \mathrm{pA}$, and $V_{\bmod }=0.05 \mathrm{mV}_{\mathrm{rms}}$. Spectroscopic measurements are done at $0.4 \mathrm{~K}$. (c) Tunneling spectra at the representative points I-IV indicated in (a). $V_{s}=+20 \mathrm{mV}, I_{t}=100 \mathrm{pA}$, and $V_{\text {mod }}=0.05 \mathrm{mV}$ rms. (d) Highresolution tunneling spectra at low $E$ taken at the same positions as for (c). $V_{s}=+10 \mathrm{mV}, I_{t}=100 \mathrm{pA}$, and $V_{\text {mod }}=0.025 \mathrm{mV}$ rms Symbols and solid lines denote experimental data and fitted results, respectively. Spectra shown in (c) and (d) are shifted vertically for clarity. (e) The exponent $\left[g(\boldsymbol{r}, E) \propto|E|^{\alpha}\right]$ determined by the fitting in the range of $|E| \leq 0.5 \mathrm{meV}$ plotted as a function of the distance from one of the TBs. The data of the single TB are shown by a red line for reference.

By contrast, line nodes along the $c$ axis are not only consistent with the strong in-plane anisotropy observed in the superconducting state, such as an elongated vortex core [6], but may also bring about nontrivial effects in the superconducting gap associated with the phase change across the TB, as we show below. Recent high-resolution laser ARPES measurements of FeSe indicate that the gap opening on the hole cylinder is isotropic in plane [30], implying that the line nodes are present on the electron cylinder. In this case, there are two possible phase structures for symmetry of the superconducting gap across a TB, as illustrated in Fig. 4, where the global phase of the superconducting gap is either fixed to the crystallographic axis [Fig. 4(a)] or flipped across the TB [Fig. 4(b)].

Note that the sign of either the nodal gap or the nodeless gap is reversed between the two domains in Figs. 4(a) or 4(b), respectively. This means that the amplitude of at least one of the gaps vanishes at the TB, giving rise to the zero-energy quasiparticle state that should appear as a zero-energy peak in $g(\boldsymbol{r}, E)$. This argument applies not only for the particular phase structure shown in Fig. 4 but also for a general case in which nodal and nodeless gaps reside on multiple Fermi surfaces.
The observed bound-state peak at $1.5 \mathrm{meV}$ apparently contradicts this conjecture and suggests instead that the TB induces an additional gap component that shifts the position of a zero-energy peak to a finite energy. We point out that, as long as the induced gap is real, a sum of the bulk gap and the TB-induced gap reverses its sign at a finite distance from the TB and still gives rise to a zero-energy peak. However, as shown in Fig. 2(b), we do not observe a zero-energy peak in $g(\boldsymbol{r}, E)$ over more than $100 \mathrm{~nm}$ from the TB. Thus, we speculate that the induced gap has an imaginary component, which means that time-reversal symmetry is locally broken near the TB. In such a case, bound state peaks are located at finite energies $E=$ $\pm \Delta \cos (\delta \varphi / 2)$ because the phase shift $\delta \varphi$ on the TB is reduced from $\pi[31,32]$. Here, $\Delta$ is the amplitude of the superconducting gap. The possibility of the TB-induced time-reversal-symmetry-broken state has been argued in $d$ wave $\mathrm{YBa}_{2} \mathrm{Cu}_{3} \mathrm{O}_{7-\delta}$ with a small $s$-wave component [33], and the splitting of the zero-energy peak has been argued theoretically [34-36]. However, the experimental observation is still lacking.

The important question here is whether this timereversal-symmetry-breaking scenario can account for the 
(a)

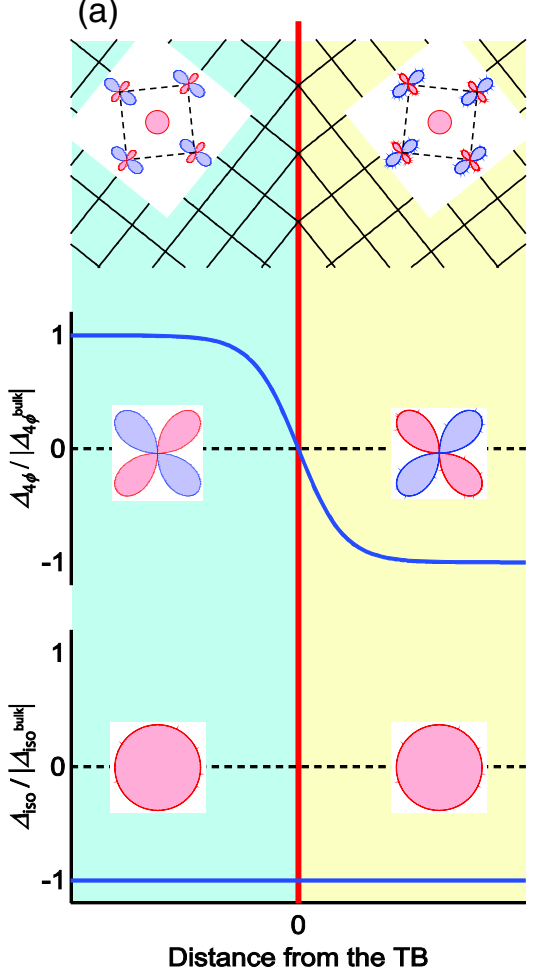

(b)

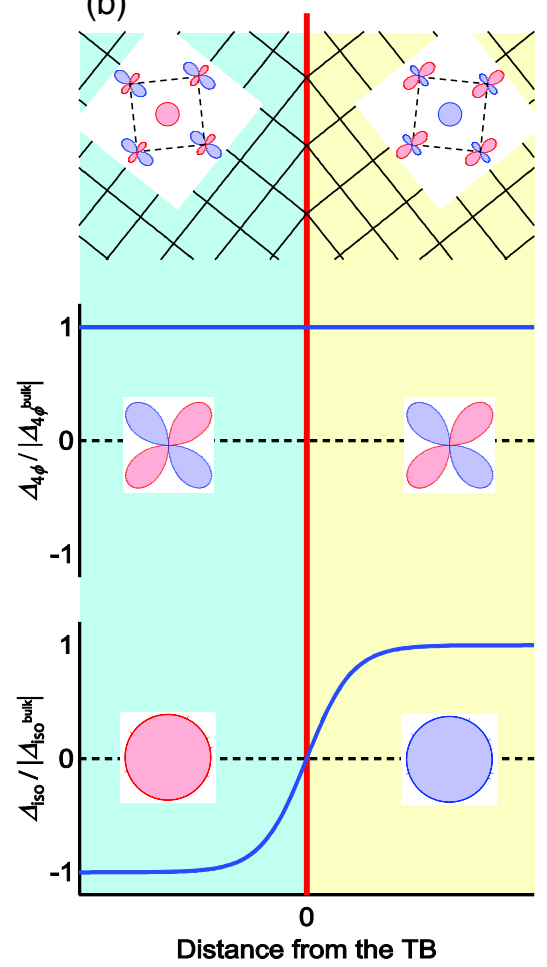

FIG. 4. Schematic illustration of the phases of the superconducting gaps across the TB shown by the red line. Top panel represents the iron lattice near the TB together with the momentum-space phase structure of the superconducting gaps opening at multiple Fermi cylinders, a hole cylinder at the center, and electron cylinders at the corner of the Brillouin zone (black broken square). Different colors (red and blue) denote different signs of the phase. We assume that the gap node exists on the electron cylinder and the sign reversal is between the main lobe of the gap on the electron cylinder and the gap on the hole cylinders, but the argument given in the text applies not only for this particular case but also for other cases. There are two possibilities: the phase structure is either fixed to the lattice (a) or flipped across the TB (b). In the former case, the nodal component $\Delta_{4 \phi}$ should change its sign across the TB, whereas the sign of the isotropic component $\Delta_{\text {iso }}$ (either due to the fully gapped Fermi cylinder or associated with the $C_{2}$ symmetry of the nodal gap) should be reversed in the latter case.

suppression of the low-energy quasiparticle excitations over a long distance from the TBs. In order to substantiate the relevance, we calculate the spatial evolution of the LDOS for a model order parameter with broken timereversal symmetry near TBs. We adopt a minimal model that includes the $C_{2}$ symmetry, the nodal gap, and the broken time-reversal symmetry near TBs. We assume that the global phase of the order parameter is fixed to the crystallographic axis; that is, the nodal component changes its sign across a TB as shown in Fig. 4(a).

The $C_{2}$-symmetric order parameter is represented by a sum of the isotropic component $\Delta_{\text {iso }}$ and the fourfold nodal component $\Delta_{4 \phi} \sin (2 \phi)$,

$$
\Delta(x)=\Delta_{\text {iso }}+\Delta_{4 \phi}(x) \sin (2 \phi),
$$

where $\phi$ is the azimuthal angle in the momentum space; see Fig. 4. The spatial variation of $\Delta_{4 \phi}$ around a TB at $x=x_{0}$ is modeled by the form

$$
\Delta_{4 \phi}(x)=\Delta_{4 \phi}^{\text {bulk }}\left\{\tanh \left[\left(x-x_{0}\right) / \xi\right] \cos \theta(x)+i \sin \theta(x)\right\},
$$

where the $x$ axis is taken to be perpendicular to the TB and $\Delta_{4 \phi}^{\text {bulk }}$ is the amplitude of $\Delta_{4 \phi}$ in the bulk. The phase $\varphi$ of $\Delta_{4 \phi}(x)$ equals $\theta(x)$ for $x-x_{0} \gg \xi$ and $\pi-\theta(x)$ for $-\left(x-x_{0}\right) \gg \xi$. The phase variable $\theta(x)$ is assumed to take a nonvanishing value near the TB and exponentially decay with another length scale $\tilde{\xi}$. It is important to note that the characteristic length $\tilde{\xi}$ for the local time-reversal symmetry breaking can be much longer than the coherence length $\xi$ [33]. (The derivation of the length $\tilde{\xi}$ is given in Appendix B.) Thus, Eq. (2) indicates the spatial variation of $\Delta_{4 \phi}$ for the amplitude over the coherence length $\xi$ and for the phase over another longer length scale $\tilde{\xi}$. To account for low-energy excitations near the nodes observed in the LDOS, we focus on the electron cylinder with nodal gaps by setting the parameters $\Delta_{\text {iso }}=0.2 \Delta_{0}$ and $\Delta_{4 \phi}^{\text {bulk }}=0.8 \Delta_{0}$.

As a model order parameter with a TB at $x=x_{0}=0$, we take $\theta(x)=(\pi / 6) \operatorname{sech}(x / \tilde{\xi})$, with $\tilde{\xi}=5 \xi$, which gives 

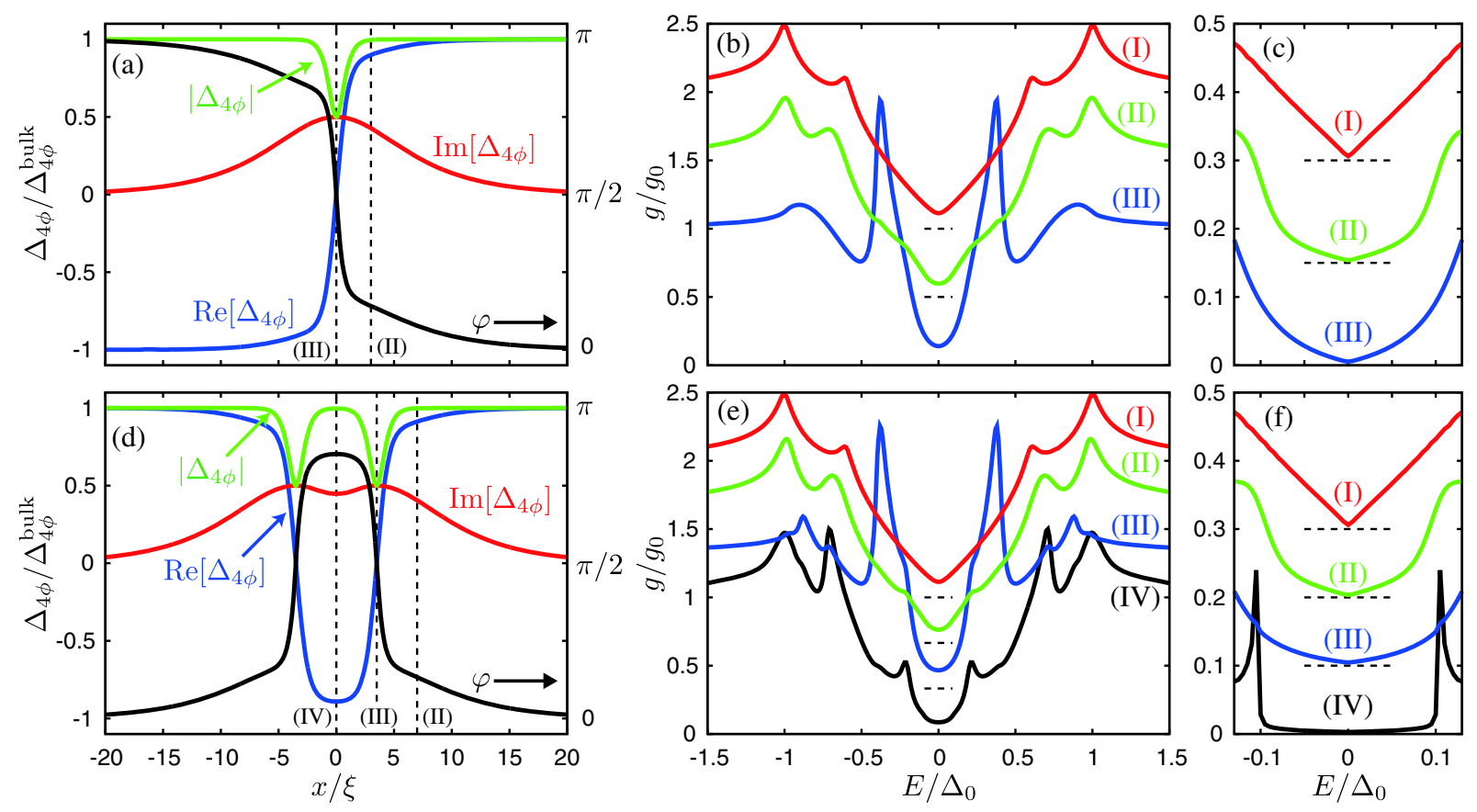

FIG. 5. (a) A model order parameter $\Delta_{4 \phi}(x)$ with a TB located at $x=0$. LDOS in the bulk (I), at $x=3 \xi$ (II), and on a TB (III), which are calculated with an energy smearing of $\eta=0.03 \Delta_{0}$ (b) and $\eta=0.001 \Delta_{0}$ (c). The lines (I) and (II) have offsets $g_{0}$ and $0.5 g_{0}$ in (b) and $0.3 g_{0}$ and $0.15 g_{0}$ in (c), respectively, where $g_{0}$ is the density of states in the normal state at the Fermi energy. (d) A model order parameter $\Delta_{4 \phi}(x)$ with double TBs located at $x= \pm 3.5 \xi$. The LDOS in the bulk (I), at $x=7 \xi$ (II), on a TB (III), and at the middle point between double TBs (IV), which are calculated with $\eta=0.03 \Delta_{0}$ (e) and $\eta=0.001 \Delta_{0}$ (f). The lines (I), (II), and (III) have offsets $g_{0}$, (2/3) $g_{0}$, and $(1 / 3) g_{0}$ in (e) and $0.3 g_{0}, 0.2 g_{0}$, and $0.1 g_{0}$, in (f).

$\Delta_{4 \phi}(x=0)=(i / 2) \Delta_{4 \phi}^{\text {bulk. }}$. The order parameter $\Delta_{4 \phi}(x)$ is shown in Fig. 5(a), where $\left|\Delta_{4 \phi}\right|$ changes with the length scale $\xi$ while $\operatorname{Im}\left(\Delta_{4 \phi}\right)$ decays with the longer length scale $\tilde{\xi}$. The phase $\varphi$ abruptly changes near the TB and gradually approaches 0 or $\pi$. Using this order parameter, we calculate the spatial dependence of the LDOS within the quasiclassical approximation [37]. Figure 5(b) shows the global peak structure of the LDOS at representative points calculated with energy smearing of $\eta=0.03 \Delta_{0}$. Far from the TB, namely in the bulk (I), the LDOS has peaks at $|E|=\Delta_{4 \phi}^{\text {bulk }} \pm \Delta_{\text {iso. }}$. On the TB (III), the peaks observed in the bulk are suppressed, and alternative peaks appear at $E \approx \pm 0.4 \Delta_{0}$, which correspond to the bound states whose energies are shifted from $E=0$ due to the local timereversal symmetry breaking in $\Delta_{4 \phi}$. The bound-state peaks disappear at $x=3 \xi$ (II) since their wave functions decay into the bulk with the length scale $\xi$. These features of the calculated LDOS arising from the bound states are consistent with the LDOS peaks observed at $E \approx \pm 1.5 \mathrm{meV}$ by STM. In Fig. 5(c) we show the LDOS at lower energy scale, which has been calculated with a much smaller smearing factor $\eta=0.001 \Delta_{0}$. The clear V-shaped LDOS in the bulk (I) changes to the U-shaped LDOS upon approaching the $\mathrm{TB}$, in agreement with the increase of the exponent $\alpha$ evaluated from the experimental data [Fig. 2(e)]. The lowenergy LDOS is finite at $x=0$ (III) and $x=3 \xi$ (II) because low-energy quasiparticles with momenta along the nodal directions of the bulk gap can linger over long distance and reach the TB, even though the local gap,

$$
|\Delta(x)|=\sqrt{\left[\Delta_{\text {iso }}+\operatorname{Re}\left(\Delta_{4 \phi}\right) \sin (2 \phi)\right]^{2}+\left[\operatorname{Im}\left(\Delta_{4 \phi}\right) \sin (2 \phi)\right]^{2}},
$$

does not vanish near the TB where $\operatorname{Im}\left(\Delta_{4 \phi}\right) \neq 0$.

We also calculate the LDOS for double TBs located at $x= \pm x_{0}= \pm 3.5 \xi$, taking the model order parameter of the form

$$
\begin{aligned}
\Delta_{4 \phi}(x)= & \Delta_{4 \phi}^{\text {bulk }}\left\{\tanh \left[\left(x-x_{0}\right) / \xi\right] \tanh \left[\left(x+x_{0}\right) / \xi\right] \cos \theta(x)\right. \\
& +i \sin \theta(x)\}
\end{aligned}
$$

shown in Fig. 5(d). We assume that the distance $2 x_{0}$ between the TBs is in the range $\xi \ll x_{0} \lesssim \tilde{\xi}$. The phase $\theta(x)$ is an even function of $x$ and takes a maximum value at $x=0$. The global peak structure of the LDOS and its lowenergy blowup are shown for representative points along the $x$ direction in Figs. 5(e) and 5(f), respectively. The large peaks at $|E| \approx 0.4 \Delta_{0}$ on a TB (III) and the small peaks at $|E| \approx 0.2 \Delta_{0}$ at the middle point $x=0$ between the TBs (IV) in Fig. 5(e) originate from the same dispersive mode of bound states at a TB. The calculated LDOS spectrum at 
$x=0$ between the double TBs (IV) in Fig. 5(f) exhibits a clear energy gap extending over the region $|E| \lesssim 0.1 \Delta_{0}$, reflecting the existence of a larger local gap at $x=0$, where the bulk low-energy quasiparticles cannot reach. We conclude that the local gap enhanced by the local time-reversal symmetry breaking near TBs over the length scale $\tilde{\xi}$ can explain the strong suppression of the LDOS between the two TBs observed in our STM or STS experiments.

\section{SUMMARY}

We report on the visualization of the atomic scale variation of the quasiparticle states of the nodal superconductor FeSe near TBs that enforce a sign inversion of the superconducting gap. In contrast to the expectation that the sign inversion generates a zero-energy quasiparticle bound state near the TB, the TB-induced quasiparticle states are not at zero but at finite energies $E \approx \pm 1.5 \mathrm{meV}$. Moreover, the low-energy excitation spectrum is affected by the TB over an extremely long distance, which is a few tens of times larger than $\xi_{a b}$. An even more dramatic change in the low-energy spectrum is detected in the region between double TBs separated by a distance $\approx 7 \xi_{a b}$, where the quasiparticle weight near the Fermi energy is almost completely removed in the energy range $|E| \lesssim 0.2 \mathrm{meV}$. These observations are qualitatively reproduced by a phenomenological model that assumes that the TB induces locally a superconducting state that breaks time-reversal symmetry.

Our results suggest several important directions for future studies. Although several models that predict timereversal-symmetry-broken superconducting state $[33,38-$ 40] have been proposed, the microscopic mechanisms and the relevance to the TB in FeSe should be clarified by future theoretical studies. It is also interesting to go beyond the quasiclassical approximation because FeSe is a unique superconductor whose Fermi energy is of the same order as the superconducting gap, placing this system in the crossover regime between Bardeen-Cooper-Schrieffer (BCS) and Bose-Einstein condensation (BEC) limits [7]. Experiments that directly probe the time-reversal symmetry breaking, such as muon-spin rotation and local magnetometry, are highly desirable and would give us further insights into the unconventional superconducting junctions. We anticipate that the $\mathrm{TBs}$ in $\mathrm{FeSe}$ will stimulate further research on the role of the phase of the superconducting order parameter near the interface, which has been difficult to access experimentally.

\section{ACKNOWLEDGMENTS}

This work has been supported by the Japan-Germany Research Cooperative Program, KAKENHI from JSPS (Grants No. 24340089 and No. 25220710), Project No. 56393598 from DAAD, and KAKENHI on Innovative Areas from MEXT of Japan (Grants No. 25103713 and No. 15H05852).

\section{APPENDIX A: ABSENCE OF LATTICE DISTORTION INDUCED BY THE TWIN BOUNDARY}

Although STM has a high spatial resolution, possible creep in the piezoelectric scanner and/or the thermal drift make it difficult to estimate the small distortions in the topographic image. Here, we utilize the so-called LawlerFujita algorithm [41] to deduce the lattice distortion and show that the TB-induced strain is negligibly small.

First, we briefly explain the principle of the methodology. The observed STM topography $T(\boldsymbol{r})$, which mainly represents the topmost Se lattice, can be expressed as

$$
\begin{aligned}
T(\boldsymbol{r})= & T_{0}\left(\cos \left\{\boldsymbol{q}_{x} \cdot[\boldsymbol{r}-\boldsymbol{u}(\boldsymbol{r})]\right\}\right. \\
& \left.+\cos \left\{\boldsymbol{q}_{y} \cdot[\boldsymbol{r}-\boldsymbol{u}(\boldsymbol{r})]\right\}\right)+\cdots .
\end{aligned}
$$

Here, $T_{0}$ is the amplitude of the Se-lattice modulation, $\boldsymbol{q}_{x}$ and $\boldsymbol{q}_{y}$ are wave vectors for the Se lattice, and center dots represent all other modulations. The distortions from the perfect lattice are described by the displacement field $\boldsymbol{u}(\boldsymbol{r})$ that can be regarded as a spatially varying phase of the $\boldsymbol{q}_{x}$ and $\boldsymbol{q}_{y}$ modulations. This approximation is justified as long as the length scale of distortions is much longer than the SeSe distance $a_{\mathrm{Se}}$. Standard phase-sensitive detection scheme can be used to evaluate $\boldsymbol{u}(\boldsymbol{r})$. By multiplying $T(\boldsymbol{r})$ and the reference signal $\cos \left(\boldsymbol{q}_{x} \cdot \boldsymbol{r}\right)$, we get

$$
\begin{aligned}
T(\boldsymbol{r}) \cos \left(\boldsymbol{q}_{x} \cdot \boldsymbol{r}\right)= & \frac{T_{0}}{2}\left\{\cos \left[\boldsymbol{q}_{x} \cdot \boldsymbol{u}(\boldsymbol{r})\right]\right. \\
& +\cos \left[2 \boldsymbol{q}_{x} \cdot \boldsymbol{r}-\boldsymbol{q}_{x} \cdot \boldsymbol{u}(\boldsymbol{r})\right] \\
& +\cos \left[\left(\boldsymbol{q}_{x}+\boldsymbol{q}_{y}\right) \cdot \boldsymbol{r}-\boldsymbol{q}_{y} \cdot \boldsymbol{u}(\boldsymbol{r})\right] \\
& \left.+\cos \left[\left(-\boldsymbol{q}_{x}+\boldsymbol{q}_{y}\right) \cdot \boldsymbol{r}-\boldsymbol{q}_{y} \cdot \boldsymbol{u}(\boldsymbol{r})\right]\right\} \\
& +\cdots .
\end{aligned}
$$

All terms except the first exhibit periodic spatial modulations, which can be removed by low-pass Fourier filtering $(\operatorname{LPF}\{\cdots\})$ :

$$
\operatorname{LPF}\left\{T(\boldsymbol{r}) \cos \left(\boldsymbol{q}_{x} \cdot \boldsymbol{r}\right)\right\}=\frac{T_{0}}{2} \cos \left[\boldsymbol{q}_{x} \cdot \boldsymbol{u}(\boldsymbol{r})\right] .
$$

By using the quadrature reference $\sin \left(\boldsymbol{q}_{x} \cdot \boldsymbol{r}\right)$, we get

$$
\operatorname{LPF}\left\{T(\boldsymbol{r}) \sin \left(\boldsymbol{q}_{x} \cdot \boldsymbol{r}\right)\right\}=\frac{T_{0}}{2} \sin \left[\boldsymbol{q}_{x} \cdot \boldsymbol{u}(\boldsymbol{r})\right] .
$$

Therefore, we obtain $u_{x}(\boldsymbol{r})$, the $x$ component of $\boldsymbol{u}(\boldsymbol{r})$, as

$$
u_{x}(\boldsymbol{r})=\frac{a_{\mathrm{Se}}}{2 \pi} \tan ^{-1} \frac{\mathrm{LPF}\left\{T(\boldsymbol{r}) \sin \left(\boldsymbol{q}_{x} \cdot \boldsymbol{r}\right)\right\}}{\operatorname{LPF}\left\{T(\boldsymbol{r}) \cos \left(\boldsymbol{q}_{x} \cdot \boldsymbol{r}\right)\right\}} .
$$



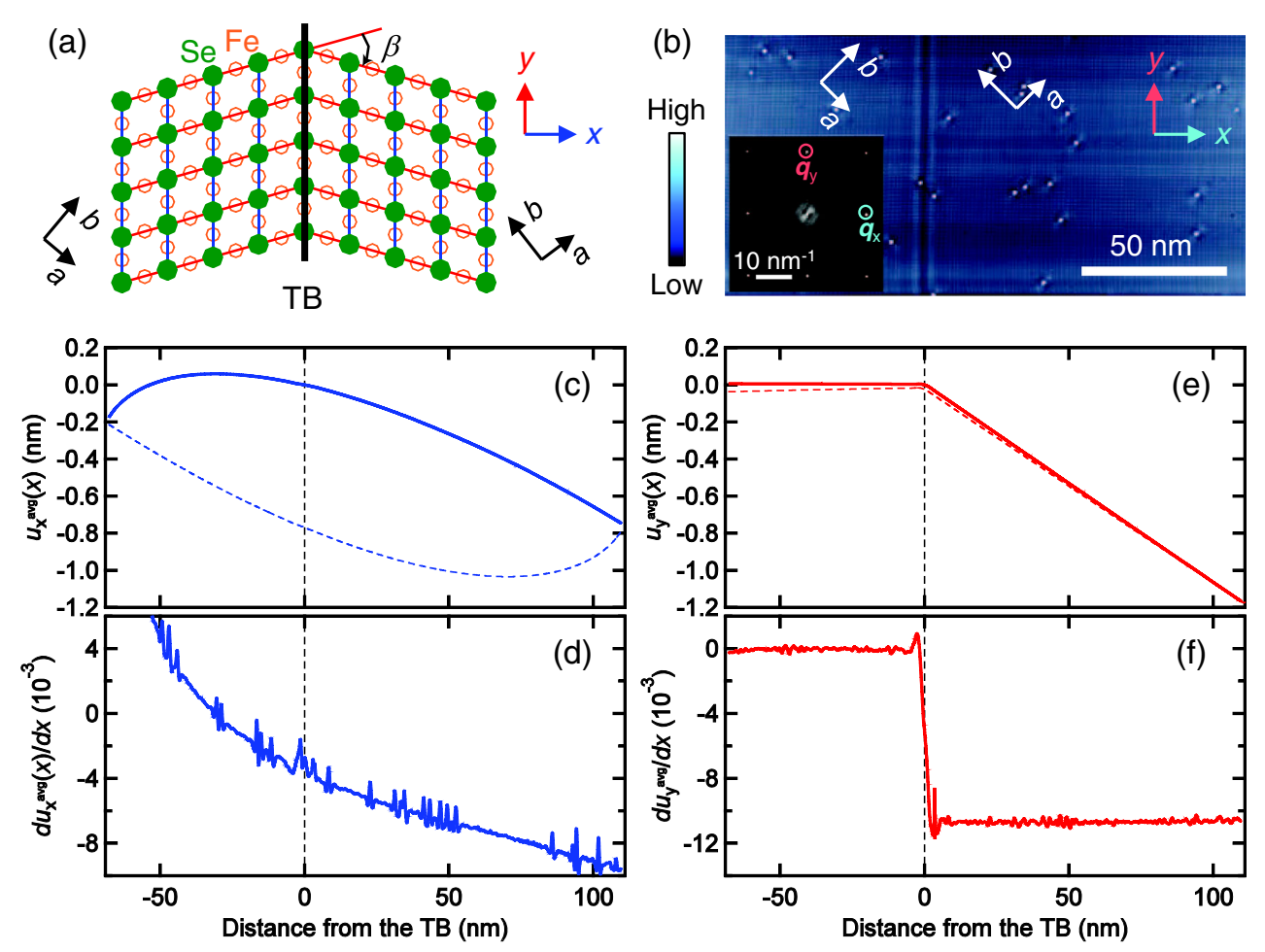

FIG. 6. (a) Schematic top view of the atomic arrangement near the TB of FeSe (not in scale). Note that an atomic periodicity along the $x$ direction is hardly affected by the TB. (b) A constant-current STM image taken over a field of view of $180 \times 90 \mathrm{~nm}^{2}$ on a grid of $4096 \times 2048$ pixels. The setup conditions for imaging are $V_{s}=+95 \mathrm{mV}$ and $I_{t}=100 \mathrm{pA}$. Inset: Fourier-transformed STM image taken in the left domain of the main panel. A peak at $\boldsymbol{q}_{x}$ is sharp and well isolated from other features, guaranteeing that $\boldsymbol{q}_{x} \cdot \boldsymbol{u}(\boldsymbol{r})$ can be treated as a spatially varying phase of the $\boldsymbol{q}_{x}$ modulations. (The same is true for $\boldsymbol{q}_{y}$.) (c) The $x$ component of $\boldsymbol{u}(\boldsymbol{r})$ averaged over the $y$ axis. Thick solid line and thin dashed line denote the data taken by the forward (left to right) and backward (right to left) scans, respectively. The symmetric hysteretic behavior between the forward and backward scans indicates that $u_{x}^{\mathrm{avg}}(x)$ is governed by the creep of the scanner. No anomaly is observed at the TB. (d) The $x$ derivative of $u_{x}^{\text {avg }}(x)$. Spikelike features are associated with the point defects in the image. (e) The $y$ component of $\boldsymbol{u}(\boldsymbol{r})$ averaged over the $y$ axis. Thick solid line and thin dashed line denote the data taken by the forward (left to right) and backward (right to left) scans, respectively. Since the $y$ direction is the slow-scan direction, the effect of the creep is small. A clear kink is observed at the TB. (f) The $x$ derivative of $u_{y}^{\text {avg }}(x)$.

The $y$ component $u_{y}(\boldsymbol{r})$ can also be deduced as

$$
u_{y}(\boldsymbol{r})=\frac{a_{\mathrm{Se}}}{2 \pi} \tan ^{-1} \frac{\operatorname{LPF}\left\{T(\boldsymbol{r}) \sin \left(\boldsymbol{q}_{y} \cdot \boldsymbol{r}\right)\right\}}{\operatorname{LPF}\left\{T(\boldsymbol{r}) \cos \left(\boldsymbol{q}_{y} \cdot \boldsymbol{r}\right)\right\}} .
$$

A schematic model of atomic arrangement near the TB is shown in Fig. 6(a). We expect that the orthorhombic distortion affects the atomic arrangement along the $y$ direction across the $\mathrm{TB}$, while the periodicity along the $x$ direction remains intact. In order to verify this model and to check if there is an additional lattice distortion, we calculate $u_{x}(\boldsymbol{r})$ and $u_{y}(\boldsymbol{r})$ of the high-resolution STM image containing a TB running along the $y$ direction [Fig 6(b)]. Reference wave vectors $\boldsymbol{q}_{x}$ and $\boldsymbol{q}_{y}$ are obtained by Fourier analysis in the left domain. For low-pass Fourier filtering, we pick up only long-wavelength components by using a Gaussian mask with half width at the half maximum of $0.21\left(2 \pi / a_{\mathrm{Se}}\right)$. Since there is a translational symmetry along the TB, we average $u_{x}(\boldsymbol{r})$ and $u_{y}(\boldsymbol{r})$ along the $y$ direction, yielding $u_{x}^{\text {avg }}(x)$ and $u_{y}^{\text {avg }}(x)$, respectively. This significantly enhances the signal-to-noise ratio.

Figures 6(c) and 6(d) show $u_{x}^{\text {avg }}(x)$ and its $x$ derivative. There is no noticeable anomaly in $u_{x}^{\text {avg }}(x)$ and $d u_{x}^{\text {avg }}(x) / d x$, except for the smooth background associated with the creep of the piezoelectric scanner. This is consistent with the model shown in Fig. 6(a) and indicates that the in-plane linear strain, which is known to affect $T_{c}$ [26], should be smaller than our detection limit of $\approx 0.5 \times 10^{-3}$. As the elastic constant $C_{11}$ is estimated to be $\approx 95 \mathrm{GPa}$ [42], the TB-induced stress, if it exists, should be smaller than $\approx 0.05 \mathrm{GPa}$, which corresponds to the change in $T_{c}$ of only $\approx 1 \%$.

By contrast, $u_{y}^{\text {avg }}(x)$ exhibits a sharp kink at the TB [Fig. 6(e)], again being consistent with the model shown in Fig. 6(a). It should be noted that $d u_{y}^{\text {avg }}(x) / d x$ shown in Fig. 6(f) is almost completely constant in both domains, indicating that the TB-induced shear strain to the lattice is also negligibly small. The observed value of $d u_{y}^{\text {avg }}(x) / d x \approx$ $-1.1 \times 10^{-2}$ in the right domain means that the angle $\beta$ 
defined in Fig. 6(a) is $+0.63^{\circ}$. This means that orthorhombic distortion $(b-a) /(b+a) \approx 2.8 \times 10^{-3}$, which is consistent with the $\mathrm{x}$-ray result [43].

\section{APPENDIX B: ASYMPTOTIC FORMS OF THE ORDER PARAMETER DERIVED BY THE GINZBURG-LANDAU THEORY}

We derive asymptotic forms of the order parameter far from TBs using the Ginzburg-Landau (GL) theory. We consider the GL free-energy functional for tetragonal symmetric systems [33] as an expansion in the isotropic $s$-wave component $\Delta_{\text {iso }}$ and the fourfold $d$-wave component $\Delta_{4 \phi}$ of the order parameter:

$$
\begin{aligned}
F_{\mathrm{GL}}\left[\Delta_{\text {iso }}, \Delta_{4 \phi}\right] & \\
= & \int d V\left\{\sum_{\mu=\text { iso }, 4 \phi}\left[\tilde{a}_{\mu}(T)\left|\Delta_{\mu}\right|^{2}+b_{\mu}\left|\Delta_{\mu}\right|^{4}+K_{\mu}\left|\nabla \Delta_{\mu}\right|^{2}\right]\right. \\
& +\gamma_{1}\left|\Delta_{\text {iso }}\right|^{2}\left|\Delta_{4 \phi}\right|^{2}+\frac{\gamma_{2}}{2}\left(\Delta_{\text {iso }}^{* 2} \Delta_{4 \phi}^{2}+\Delta_{\text {iso }}^{2} \Delta_{4 \phi}^{* 2}\right) \\
& \left.+\frac{\tilde{K}}{2}\left[\left(\partial_{a} \Delta_{\text {iso }}\right)^{*}\left(\partial_{a} \Delta_{4 \phi}\right)-\left(\partial_{b} \Delta_{\text {iso }}\right)^{*}\left(\partial_{b} \Delta_{4 \phi}\right)+\text { c.c. }\right]\right\},
\end{aligned}
$$

where we have neglect the vector potential as it does not play an important role in our discussion. The coefficients $b_{\mu}, K_{\mu}$, and $\tilde{K}$ are positive, and $\tilde{a}_{\mu}(T)=a_{\mu}\left(T / T_{\mathrm{c} \mu}-1\right)$ with positive $a_{\mu}$. The differential operator $\boldsymbol{\nabla}=\left(\partial_{a}, \partial_{b}\right)$ is defined according to the crystal axes $a$ and $b$. As in Ref. [33], we assume $\gamma_{2}>0$, so that the free energy is minimized at $\varphi= \pm \pi / 2$, and the time-reversal-symmetrybroken $s \pm i d$ state is stabilized when both $\Delta_{\text {iso }}$ and $\Delta_{4 \phi}$ are finite.

The effect of orthorhombic distortion is taken into account by adding the following term to the free-energy functional [33]:

$$
F_{\epsilon}=c \epsilon \int d V\left(\Delta_{\text {iso }}^{*} \Delta_{4 \phi}+\Delta_{\text {iso }} \Delta_{4 \phi}^{*}\right),
$$

where $c$ is a positive parameter and $\epsilon=\epsilon_{a a}-\epsilon_{b b}$ is the parameter of the orthorhombic lattice distortion. The total free energy for a uniform state in the bulk is then given by

$$
\begin{aligned}
\frac{F_{\mathrm{GL}}+F_{\epsilon}}{V}= & \sum_{\mu=\text { iso }, 4 \phi}\left(\tilde{a}_{\mu}\left|\Delta_{\mu}\right|^{2}+b_{\mu}\left|\Delta_{\mu}\right|^{4}\right)+\gamma_{1}\left|\Delta_{\text {iso }}\right|^{2}\left|\Delta_{4 \phi}\right|^{2} \\
& +\gamma_{2}\left|\Delta_{\text {iso }}\right|^{2}\left|\Delta_{4 \phi}\right|^{2} \cos (2 \varphi) \\
& +2 c \epsilon\left|\Delta_{\text {iso }}\right|\left|\Delta_{4 \phi}\right| \cos \varphi
\end{aligned}
$$

where $\Delta_{\mu}=\left|\Delta_{\mu}\right| e^{i \varphi_{\mu}}$ and the relative phase $\varphi=\varphi_{4 \phi}-\varphi_{\text {iso }}$. If $c|\epsilon| \geq 2 \gamma_{2}\left|\Delta_{\text {iso }}\right|\left|\Delta_{4 \phi}\right|$, then the free energy is minimized at $\varphi=0$ for $\epsilon<0$ and at $\varphi=\pi$ for $\epsilon>0$. In the following discussion, we assume that this inequality is satisfied and the time-reversal symmetric $s \pm d$ state is realized in the bulk.

Next, we consider a TB located at $x=x_{0}$ along the $y$ axis, where the $x$ and $y$ axes are rotated by $45^{\circ}$ from the crystalline axes, $x=(a-b) / \sqrt{2}$ and $y=(a+b) / \sqrt{2}$. The orthorhombic lattice distortion parameter $\epsilon$ changes its sign across the TB. We assume $\epsilon(x) \rightarrow \mp|\epsilon|$ for $x \rightarrow \pm \infty$, so that the $s \pm d$ state is realized in $x \rightarrow \pm \infty$. Near the TB where $\epsilon(x)$ is small, the $s \pm i d$ state is favored. Then, the area density of the total free energy is given by

$$
\begin{aligned}
f_{\mathrm{GL}}+f_{\epsilon}= & \int d x\left[\sum_{\mu=\mathrm{iso}, 4 \phi}\left(\tilde{a}_{\mu}\left|\Delta_{\mu}\right|^{2}+b_{\mu}\left|\Delta_{\mu}\right|^{4}+K_{\mu}\left|\partial_{x} \Delta_{\mu}\right|^{2}\right)\right. \\
& +\gamma_{1}\left|\Delta_{\mathrm{iso}}\right|^{2}\left|\Delta_{4 \phi}\right|^{2}+\frac{\gamma_{2}}{2}\left(\Delta_{\mathrm{iso}}^{* 2} \Delta_{4 \phi}^{2}+\Delta_{\mathrm{iso}}^{2} \Delta_{4 \phi}^{* 2}\right) \\
& \left.+c \epsilon(x)\left(\Delta_{\mathrm{iso}}^{*} \Delta_{4 \phi}+\Delta_{\text {iso }} \Delta_{4 \phi}^{*}\right)\right] .
\end{aligned}
$$

We first assume that $\Delta_{\text {iso }}$ is a real and uniform order parameter while $\Delta_{4 \phi}$ changes its sign across the TB, as shown in Fig. 4(a). If we restrict $\Delta_{4 \phi}$ to be real, then $\Delta_{4 \phi}$ varies over the coherence length [33],

$$
\xi=\sqrt{\frac{K_{4 \phi}}{\tilde{a}_{4 \phi}+6 b_{4 \phi}\left|\Delta_{4 \phi}^{\text {bulk }}\right|^{2}+\left(\gamma_{1}+\gamma_{2}\right)\left|\Delta_{\text {iso }}\right|^{2}}},
$$

where $\left|\Delta_{4 \phi}^{\text {bulk }}\right|$ is the amplitude of $\Delta_{4 \phi}$ in the bulk. However, we expect that time-reversal symmetry should be locally broken at the TB. Thus, we allow $\Delta_{4 \phi}$ to be complex, $\Delta_{4 \phi}(x)=\left|\Delta_{4 \phi}(x)\right| e^{i \varphi(x)}$. With this order parameter, the total free energy is given by

$$
\begin{aligned}
f_{\mathrm{GL}}+f_{\epsilon}= & \int d x\left[\tilde{a}_{\text {iso }}\left|\Delta_{\text {iso }}\right|^{2}+b_{\text {iso }}\left|\Delta_{\text {iso }}\right|^{4}+\tilde{a}_{4 \phi}\left|\Delta_{4 \phi}\right|^{2}\right. \\
& +b_{4 \phi}\left|\Delta_{4 \phi}\right|^{4}+\gamma_{1}\left|\Delta_{\text {iso }}\right|^{2}\left|\Delta_{4 \phi}\right|^{2} \\
& +\gamma_{2}\left|\Delta_{\text {iso }}\right|^{2}\left|\Delta_{4 \phi}\right|^{2} \cos (2 \varphi) \\
& +2 c \epsilon\left(x-x_{0}\right)\left|\Delta_{\text {iso }}\right|\left|\Delta_{4 \phi}\right| \cos \varphi \\
& \left.+K_{4 \phi}\left[\left(\partial_{x}\left|\Delta_{4 \phi}\right|\right)^{2}+\left|\Delta_{4 \phi}\right|^{2}\left(\partial_{x} \varphi\right)^{2}\right]\right] .
\end{aligned}
$$

In the bulk region $\left(x-x_{0} \gg \xi\right)$, where $\partial_{x}\left|\Delta_{4 \phi}\right|=0$ and $\epsilon(x)=-|\epsilon|$, the GL differential equation to minimize $f_{\mathrm{GL}}+f_{\epsilon}$ is

$$
K_{4 \phi} \partial_{x}^{2} \varphi=-\frac{\left|\Delta_{\text {iso }}\right|}{\left|\Delta_{4 \phi}^{\text {bulk }}\right|}\left[\gamma_{2}\left|\Delta_{\text {iso }}\right|\left|\Delta_{4 \phi}^{\text {bulk }}\right| \sin (2 \varphi)-c|\epsilon| \sin \varphi\right] .
$$

Since $\varphi \ll 1$ far away from the TB, we can linearize the differential equation and find the relative phase to decay as $\varphi \propto \exp (-x / \tilde{\xi})$ with the characteristic length 


$$
\tilde{\xi}=\sqrt{\frac{K_{4 \phi}\left|\Delta_{4 \phi}^{\text {bulk }}\right|}{\left|\Delta_{\text {iso }}\right|\left(c|\epsilon|-2 \gamma_{2}\left|\Delta_{\text {iso }}\right|\left|\Delta_{4 \phi}^{\text {bulk }}\right|\right)}}
$$

The characteristic length diverges when approaching the phase boundary, where $c|\epsilon|=2 \gamma_{2}\left|\Delta_{\text {iso }}\right|\left|\Delta_{4 \phi}\right|$, between the time-reversal symmetric $s \pm d$ state and the time-reversalsymmetry-broken $s \pm i d$ state.

Finally, we consider double TBs at $x= \pm\left|x_{0}\right|$, where $\xi \ll\left|x_{0}\right| \lesssim \tilde{\xi}$. We assume $\epsilon>0$ between the double TBs and $\epsilon<0$ otherwise. At the center $x=0$ between the double TBs, we can set $\left|\Delta_{4 \phi}\right|=\left|\Delta_{4 \phi}^{\text {bulk }}\right|$ because $\left|x_{0}\right| \gg \xi$. With this approximation, the GL differential equation to minimize $f_{\mathrm{GL}}+f_{\epsilon}$ for $|x| \ll\left|x_{0}\right|$ is

$K_{4 \phi} \partial_{x}^{2} \varphi=-\frac{\left|\Delta_{\text {iso }}\right|}{\left|\Delta_{4 \phi}^{\text {bulk }}\right|}\left[\gamma_{2}\left|\Delta_{\text {iso }}\right|\left|\Delta_{4 \phi}^{\text {bulk }}\right| \sin (2 \varphi)+c|\epsilon| \sin \varphi\right]$.

Integration of the differential equation yields

$$
\begin{aligned}
K_{4 \phi}\left(\partial_{x} \varphi\right)^{2}= & \frac{\left|\Delta_{\text {iso }}\right|}{\left|\Delta_{4 \phi}^{\text {bulk }}\right|}\left[\gamma_{2}\left|\Delta_{\text {iso }}\right|\left|\Delta_{4 \phi}^{\text {bulk }}\right| \cos (2 \varphi)+2 c|\epsilon| \cos \varphi\right. \\
& \left.-\gamma_{2}\left|\Delta_{\text {iso }}\right|\left|\Delta_{4 \phi}^{\text {bulk }}\right| \cos \left(2 \varphi_{0}\right)-2 c|\epsilon| \cos \varphi_{0}\right],
\end{aligned}
$$

where the integration constant is determined from the conditions $\partial_{x} \varphi(x=0)=0$ and $\varphi(x=0) \equiv \varphi_{0}$. Since we assume the distance between the TBs is in the range $\left|x_{0}\right| \lesssim \tilde{\xi}$, the relative phase does not reach $\pi$ at $x=0$; i.e., $\varphi_{0}<\pi$. For $\varphi_{0}-\varphi \ll 1$ near $x=0$, the differential equation [Eq. (B10)] has the solution

$$
\varphi(x)=\varphi_{0}-\left(\frac{x}{\tilde{\xi}_{0}}\right)^{2} .
$$

For the model order parameter shown in Fig. 5(d), we determine $\varphi_{0}$ and $\tilde{\xi}_{0}$ by the continuity condition at $x= \pm\left|x_{0}\right| / 2$, that is, by imposing that $\varphi=\pi-$ $(\pi / 6) \operatorname{sech}(x / \tilde{\xi})$ and $\varphi(x)$ in Eq. (B11) are smoothly connected at $x= \pm\left|x_{0}\right| / 2$. We note that different choices of the connecting position yield little change in the value of $\varphi$.

[1] T. Van Duzer and C. W. Turner, Principles of Superconductive Devices and Circuits, 2nd ed. (Prentice-Hall, Englewood Cliffs, NJ, 1998).

[2] C. C. Tsuei and J. R. Kirtley, Pairing Symmetry in Cuprate Superconductors, Rev. Mod. Phys. 72, 969 (2000).

[3] J. Kim, V. Chua, G. A. Fiete, H. Nam, A. H. MacDonald, and C.-K. Shih, Visualization of Geometric Influences on
Proximity Effects in Heterogeneous Superconductor Thin Films, Nat. Phys. 8, 464 (2012).

[4] L. Serrier-Garcia, J. C. Cuevas, T. Cren, C. Brun, V. Cherkez, F. Debontridder, D. Fokin, F. S. Bergeret, and D. Roditchev, Scanning Tunneling Spectroscopy Study of the Proximity Effect in a Disordered Two-Dimensional Metal, Phys. Rev. Lett. 110, 157003 (2013).

[5] V. Cherkez, J. C. Cuevas, C. Brun, T. Cren, G. Menard, F. Debontridder, V. S. Stolyarov, and D. Roditchev, Proximity Effect between Two Superconductors Spatially Resolved by Scanning Tunneling Spectroscopy, Phys. Rev. X 4, 011033 (2014).

[6] C.-L. Song, Y.-L. Wang, P. Cheng, Y.-P. Jiang, W. Li, T. Zhang, Z. Li, K. He, L. Wang, J.-F. Jia, H.-H. Hung, C. Wu, X. Ma, X. Chen, and Q.-K. Xue, Direct Observation of Nodes and Twofold Symmetry in FeSe Superconductor, Science 332, 1410 (2011).

[7] S. Kasahara, T. Watashige, T. Hanaguri, Y. Kohsaka, T. Yamashita, Y. Shimoyama, Y. Mizukami, R. Endo, H. Ikeda, K. Aoyama, T. Terashima, S. Uji, T. Wolf, H. v. Löhneysen, T. Shibauchi, and Y. Matsuda, Field-Induced Superconducting Phase of FeSe in the BCS-BEC Cross-Over, Proc. Natl. Acad. Sci. U.S.A. 111, 16309 (2014).

[8] D. J. Derro, S. H. Pan, E. W. Hudson, K. M. Lang, J. C. Davis, K. Mochizuki, J. T. Markert, and A. de Lozanne, A Detailed Scanning Tunneling Microscopy Study of the CuO Chains of $\mathrm{YBa}_{2} \mathrm{Cu}_{3} \mathrm{O}_{7-x}$, Physica (Amsterdam) 341-348C, 425 (2000).

[9] T.-M. Chuang, M. P. Allan, J. Lee, Y. Xie, N. Ni, S. L. Bud'ko, G. S. Boebinger, P. C. Canfield, and J. C. Davis, Nematic Electronic Structure in the "Parent" State of the Iron-Based Superconductor $\mathrm{Ca}\left(\mathrm{Fe}_{1-x} \mathrm{Co}_{x}\right)_{2} \mathrm{As}_{2}$, Science 327, 181 (2010).

[10] E. P. Rosenthal, E. F. Andrade, C. J. Arguello, R. M. Fernandes, L. Y. Xing, X. C. Wang, C. Q. Jin, A. J. Millis, and A. N. Pasupathy, Visualization of Electron Nematicity and Unidirectional Antiferroic Fluctuations at High Temperatures in NaFeAs, Nat. Phys. 10, 225 (2014).

[11] F.-C. Hsu, J.-Y. Luo, K.-W. Yeh, T.-K. Chen, T.-W. Huang, P. M. Wu, Y.-C. Lee, Y.-L. Huang, Y.-Y. Chu, D.-C. Yan, and M.-K. Wu, Superconductivity in the PbO-Type Structure $\alpha$-FeSe, Proc. Natl. Acad. Sci. U.S.A. 105, 14262 (2008).

[12] K. Nakayama, Y. Miyata, G. N. Phan, T. Sato, Y. Tanabe, T. Urata, K. Tanigaki, and T. Takahashi, Reconstruction of Band Structure Induced by Electronic Nematicity in an FeSe Superconductor, Phys. Rev. Lett. 113, 237001 (2014).

[13] T. Shimojima, Y. Suzuki, T. Sonobe, A. Nakamura, M. Sakano, J. Omachi, K. Yoshioka, M. Kuwata-Gonokami, K. Ono, H. Kumigashira, A. E. Böhmer, F. Hardy, T. Wolf, C. Meingast, H. v. Löhneysen, H. Ikeda, and K. Ishizaka, Lifting of $x z / y z$ Orbital Degeneracy at the Structural Transition in Detwinned FeSe, Phys. Rev. B 90, 121111 (R) (2014).

[14] M. D. Watson, T. K. Kim, A. A. Haghighirad, N. R. Davies, A. McCollam, A. Narayanan, S. F. Blake, Y. L. Chen, S. Ghannadzadeh, A. J. Schofield, M. Hoesch, C. Meingast, T. Wolf, and A. I. Coldea, Emergence of the Nematic Electronic State in FeSe, Phys. Rev. B 91, 155106 (2015). 
[15] P. Zhang, T. Qian, P. Richard, X. P. Wang, H. Miao, B. Q. Lv, B. B. Fu, T. Wolf, C. Meingast, X. X. Wu, Z. Q. Wang, J. P. Hu, and H. Ding, Observation of Two Distinct $d_{x z} / d_{y z}$ Band Splittings in FeSe, Phys. Rev. B 91, 214503 (2015).

[16] A. E. Böhmer, T. Arai, F. Hardy, T. Hattori, T. Iye, T. Wolf, H. v. Löhneysen, K. Ishida, and C. Meingast, Origin of the Tetragonal-to-Orthorhombic Phase Transition in FeSe: A Combined Thermodynamic and NMR Study of Nematicity, Phys. Rev. Lett. 114, 027001 (2015).

[17] S.-H. Baek, D. V. Efremov, J. M. Ok, J. S. Kim, Jeroen van den Brink, and B. Büchner, Orbital-Driven Nematicity in FeSe, Nat. Mater. 14, 210 (2015).

[18] K. Momma and F. Izumi, VESTA 3 for Three-Dimensional Visualization of Crystal, Volumetric and Morphology Data, J. Appl. Crystallogr. 44, 1272 (2011).

[19] A. Subedi, L. Zhang, D. J. Singh, and M. H. Du, Density Functional Study of FeS, FeSe, and FeTe: Electronic Structure, Magnetism, Phonons, and Superconductivity, Phys. Rev. B 78, 134514 (2008).

[20] M. Aichhorn, S. Biermann, T. Miyake, A. Georges, and M. Imada, Theoretical Evidence for Strong Correlations and Incoherent Metallic State in FeSe, Phys. Rev. B 82, 064504 (2010).

[21] T. Terashima, N. Kikugawa, A. Kiswandhi, E.-S. Choi, J. S. Brooks, S. Kasahara, T. Watashige, H. Ikeda, T. Shibauchi, Y. Matsuda, T. Wolf, A. E. Böhmer, F. Hardy, C. Meingast, H. v. Löhneysen, M.-T. Suzuki, R. Arita, and S. Uji, Anomalous Fermi Surface in FeSe Seen by Shubnikov-de Haas Oscillation Measurements, Phys. Rev. B 90, 144517 (2014).

[22] M. D. Watson, T. Yamashita, S. Kasahara, W. Knafo, M. Nardone, J. Beard, F. Hardy, A. McCollam, A. Narayanan, S. F. Blake, T. Wolf, A. A. Haghighirad, C. Meingast, A. J. Schofield, H. v. Löhneysen, Y. Matsuda, A. I. Coldea, and T. Shibauchi, Dichotomy between the Hole and Electron Behavior in the Multiband FeSe Probed by Ultrahigh Magnetic Fields, Phys. Rev. Lett. 115, 027006 (2015).

[23] J. Maletz, V. B. Zabolotnyy, D. V. Evtushinsky, S. Thirupathaiah, A. U. B. Wolter, L. Harnagea, A. N. Yaresko, A. N. Vasiliev, D. A. Chareev, A. E. Böhmer, F. Hardy, T. Wolf, C. Meingast, E. D. L. Rienks, B. Büchner, and S. V. Borisenko, Unusual Band Renormalization in the Simplest Iron-Based Superconductor $\mathrm{FeSe}_{1-x}$, Phys. Rev. B 89, 220506(R) (2014).

[24] C.-L. Song, Y.-L. Wang, Y.-P. Jiang, L. Wang, K. He, X. Chen, J. E. Hoffman, X.-C. Ma, and Q.-K. Xue, Suppression of Superconductivity by Twin Boundaries in FeSe, Phys. Rev. Lett. 109, 137004 (2012).

[25] T. Hanaguri, Development of High-Field STM and Its Application to the Study on Magnetically-Tuned Criticality in $\mathrm{Sr}_{3} \mathrm{Ru}_{2} \mathrm{O}_{7}$, J. Phys. Conf. Ser. 51, 514 (2006).

[26] A. E. Böhmer, F. Hardy, F. Eilers, D. Ernst, P. Adelmann, P. Schweiss, T. Wolf, and C. Meingast, Lack of Coupling between Superconductivity and Orthorhombic Distortion in Stoichiometric Single-Crystalline FeSe, Phys. Rev. B 87, 180505(R) (2013).
[27] S. Graser, A. F. Kemper, T. A. Maier, H.-P. Cheng, P. J. Hirschfeld, and D. J. Scalapino, Spin Fluctuations and Superconductivity in a Three-Dimensional Tight-Binding Model for $\mathrm{BaFe}_{2} \mathrm{As}_{2}$, Phys. Rev. B 81, 214503 (2010).

[28] K. Suzuki, H. Usui, and K. Kuroki, Possible ThreeDimensional Nodes in the $s \pm$ Superconducting Gap, J. Phys. Soc. Jpn. 80, 013710 (2011).

[29] Y. Su, C. Setty, Z. Wang, and J. Hu, c-Axis Nodal Lines Induced by Interlayer Pairing in Iron-Based Superconductors, Phys. Rev. B 85, 184517 (2012).

[30] K. Okazaki (private communication).

[31] D. Rainer, H. Burkhardt, M. Fogelström, and J. A. Sauls, Andreev Bound States, Surfaces and Subdominant Pairing in High $T_{c}$ Superconductors, J. Phys. Chem. Solids 59, 2040 (1998).

[32] A. Furusaki, Josephson Current Carried by Andreev Levels in Superconducting Quantum Point Contacts, Superlattices Microstruct. 25, 809 (1999).

[33] M. Sigrist, K. Kuboki, P. A. Lee, A. J. Millis, and T. M. Rice, Influence of Twin Boundaries on Josephson Junctions between High-Temperature and Conventional Superconductors, Phys. Rev. B 53, 2835 (1996).

[34] M. E. Zhitomirsky and M. B. Walker, Electronic States on a Twin Boundary of a d-Wave Superconductor, Phys. Rev. Lett. 79, 1734 (1997).

[35] D. L. Feder, A. Beardsall, A. J. Berlinsky, and C. Kallin, Twin Boundaries in d-Wave Superconductors, Phys. Rev. B 56, R5751 (1997).

[36] W. Belzig, C. Bruder, and M. Sigrist, Quasiclassical Theory of Twin Boundaries in High- $T_{c}$ Superconductors, Phys. Rev. Lett. 80, 4285 (1998).

[37] N. Schopohl and K. Maki, Quasiparticle Spectrum around a Vortex Line in a d-Wave Superconductor, Phys. Rev. B 52, 490 (1995).

[38] M. Matsumoto and H. Shiba, Coexistence of Different Symmetry Order Parameters near a Surface in $d$-Wave Superconductors I, J. Phys. Soc. Jpn. 64, 3384 (1995).

[39] X. Hu and Z. Wang, Stability and Josephson Effect of TimeReversal-Symmetry-Broken Multicomponent Superconductivity Induced by Frustrated Intercomponent Coupling, Phys. Rev. B 85, 064516 (2012).

[40] J. Kang, A. F. Kemper, and R. M. Fernandes, Manipulation of Gap Nodes by Uniaxial Strain in Iron-Based Superconductors, Phys. Rev. Lett. 113, 217001 (2014).

[41] M. J. Lawler, K. Fujita, J. Lee, A. R. Schmidt, Y. Kohsaka, C. K. Kim, H. Eisaki, S. Uchida, J. C. Davis, J. P. Sethna, and E.-A. Kim, Intra-Unit-Cell Electronic Nematicity of the High- $T_{c}$ Copper-Oxide Pseudogap States, Nature (London) 466, 347 (2010).

[42] S. Chandra and A. K. M. A. Islam, Elastic Properties of Mono- and Poly-Crystalline PbO-Type $\mathrm{FeSe}_{1-x} \mathrm{Te}_{x}$ $(x=0-1.0):$ A First-Principles Study, Physica (Amsterdam) 470C, 2072 (2010).

[43] R. Khasanov, M. Bendele, K. Conder, H. Keller, E. Pomjakushina, and V. Pomjakushin, Iron Isotope Effect on the Superconducting Transition Temperature and the Crystal Structure of $\mathrm{FeSe}_{1-x}$ New J. Phys. 12, 073024 (2010). 